\title{
3Rs for innovating novel antibiotics: sharing resources, risks, and rewards
}

The stream of new antibiotics is struggling to keep up with emerging bacterial resistance. Anthony So and colleagues examine what can be done to increase innovation

\author{
Anthony D So director ${ }^{1}$, Quentin Ruiz-Esparza associate in research ${ }^{1}$, Neha Gupta associate in \\ research ${ }^{1}$, Otto Cars professor of infectious diseases ${ }^{2}$
}

${ }^{1}$ Program on Global Health and Technology Access, Sanford School of Public Policy, Duke University, Durham, NC 27708, USA; ${ }^{2}$ Department of Medical Sciences, Uppsala University, Uppsala, Sweden

\begin{abstract}
The dearth of novel antibiotics poses challenges to the treatment of bacterial infection and points to shortcomings in the system of pharmaceutical innovation. Increasing bacterial resistance to existing antibiotics causes substantial morbidity and mortality and threatens society's ability to realise benefits from modern medical advances. Access to effective antibiotics is essential to treating the unavoidable infections that come with cancer chemotherapy, organ transplantation, or the care of premature babies.
\end{abstract}

Yet studies have repeatedly confirmed the faltering research pipeline for novel antibiotics and cited the exit of major pharmaceutical firms from this therapeutic area. In the publicly disclosed pipelines of the top 15 drug companies, only five drug candidates, or $1.6 \%$ of the pipeline, were antibiotics. ${ }^{1}$ A more comprehensive search of two commercial databases also turned up few novel antibacterial drug candidates. ${ }^{2}$ Of the 15 candidates identified that could be administered systemically, only four were active against Gram negative bacteria, two of which acted on new targets; none of the four had a novel mechanism of action. $^{2}$

With few promising drug candidates in sight, the near term prospects of new antibiotics are dismal. ${ }^{3}$ The bottlenecks in bringing a novel antibiotic to market span from discovery to delivery (fig $1 \Downarrow$ ). Upstream in the research and development pipeline, concerns have surfaced over identification of leads and medicinal chemistry. Especially critical is the step between preclinical and clinical development (the "valley of death"). Drug companies have been hesitant to take compounds into large and costly clinical programmes because of the uncertain return on investment and academic researchers and smaller companies find it difficult to get venture capital for clinical research. Downstream, concerns over the regulatory approval process have stirred debate, and financing research and development may also pose barriers.
Drug companies have to see that their expected returns will exceed the costs of research and development. But compared with other therapeutic categories, the economic value of antibiotics to pharmaceutical firms is considerably lower. ${ }^{4}$ Research into antibiotics therefore often loses out to potentially more lucrative health technologies.

Possible interventions to improve the pipeline have been identified. ${ }^{56}$ But identifying which is the inspired solution is not easy. Nor is there likely to be a single solution. Effective solutions are likely to include sharing the three Rs-resources, risks, and rewards..

\section{Sharing resources}

The availability of resources-particularly research inputs-is important in tackling challenges to drug discovery. Although the range of promising targets is not a primary limiting factor, existing compound libraries and the methods used to mine them have not identified sufficient drug candidates. GlaxoSmithKline garnered just five leads from 70 automated high throughput screens conducted between 1995 and 2001- a yield fourfold to fivefold lower than for other therapeutic areas.?

It is questionable whether further mining of existing libraries will ever produce more positive results. Other strategies that might increase yields include enriching collections with natural products, fragment based screening, additional work on parts of the genome thought to be less easily druggable, and structural genomics. ${ }^{8}$ Part of the problem may lie in the emphasis on "rational" drug development focused on single targets and high throughput screening. We need to get back to the basics of biology - "targeting an organism (bacterium) inside another organism (the human host)"- and give more attention to the potential of resistance arising rapidly. ${ }^{9}$

In addition, limited access to medicinal chemistry resources may hinder the development of leads. Medicinal chemistry is needed to determine the pharmacokinetic properties; 
structure-activity relationship; absorption, distribution, metabolism, and excretion; and safety profile. ${ }^{10}$ Smaller firms or academic research groups might benefit from centralised access to contracted medicinal chemistry services.

Sharing resources might allow a greater diversity of groups to search for novel antibiotics. With support from the Medicines for Malaria Venture, GlaxoSmithKline released the chemical structures and assay data for 13500 compounds it had identified as having antimalarial activity against Plasmodium falciparum. The information was deposited in the European Bioinformatics Institute's freely available ChEMBL database and the US National Institutes of Health (NIH) PubChem database. Various models have sought to broker similar access to data that remain proprietary. The European Rare Diseases Therapeutic Initiative focuses on enabling academic research teams to access proprietary compound libraries for preclinical studies of rare diseases. ${ }^{11}$ The Special Programme for Research and Training in Tropical Diseases has also secured access to the compound libraries of Merck Serono and Pfizer. ${ }^{12}$ These arrangements have provisions in common, including a layer of confidentiality, the option of first refusal, and potential access to proprietary data. Of note, similar collaborative strategies have been proposed for companies seeking access to small molecules of potential commercial value, not just those for rare or neglected diseases. ${ }^{13}$

While companies might once have balked at sharing information that could advantage competitors, the line between precompetitive and competitive data has shifted downstream, leading to unprecedented collaborations. The need for better treatments for neurodegenerative diseases, notably Alzheimer's and Parkinson's disease, led the Coalition Against Major Diseases to develop common clinical data standards and a pooled database of control groups in clinical trials from multiple companies. $^{14}$

Building the public infrastructure for compound libraries and their screening might complement access to proprietary collections. One such example is the NIH Molecular Libraries Probe Production Centers Network. ${ }^{15}$ Structural information on compounds deposited in the Molecular Libraries Small Molecule Repository and screening data generated has become publicly available in PubChem. By overcoming scientific challenges, such sharing of resources helps to reduce the risks of research and development.

\section{Sharing risks}

Sharing the risks of research and development across public and private sectors eases the transition from preclinical to clinical testing. Public sector support is already accelerating development of treatments for rare diseases. For example, in the US, the Therapeutics for Rare and Neglected Diseases programme uses NIH's intramural resources to bring drug leads forward to meet FDA requirements for an application for an investigational new drug. By contrast, the Bridging Interventional Development Gaps programme allows those developing new drugs to compete for services, contracted by the government, for formulation of good manufacturing practices, animal toxicology, and development of assays for pharmacokinetic testing. Applied to antibiotics, such services might well boost the success of preclinical research and development. NIH has opened the National Center for Advancing Translational Sciences, which will consolidate such efforts to fill research gaps. ${ }^{16}$

Disease specific, patient driven foundations have also had an important role in developing collaborative research. In the US, the Cystic Fibrosis Foundation's therapeutics development network has linked 18 national research centres. Together they have conducted over 40 clinical trials-including several on antibiotics-involving 4700 patients (more than a sixth of people with the disease in the United States). ${ }^{17}$ They have also developed improved trial protocols and standardised endpoints, driving forward the search for new cystic fibrosis treatments despite the relatively small market.

Public funding could provide a platform for innovation. India's Council for Scientific and Industrial Research provided government funding for the Open Source Drug Discovery project in which hundreds of volunteer scientists and students at universities collaborated online to re-annotate the Mycobacterium tuberculosis genome. The volunteers completed many person-years of work in just four months. ${ }^{18}$ Regional innovation platforms such as the African Network for Drugs and Diagnostics Innovation (ANDI) have also emerged, along with sister networks in Asia and America, to leverage existing research capacity and open doors to South-South collaboration. ${ }^{19}$

Government and philanthropic funding for antibiotic research is being made available to a growing range of actors. The US Department of Defense has awarded contracts to companies including GlaxoSmithKline's Antimicrobial Resistance Center for Excellence in Drug Discovery and Trius Therapeutics. ${ }^{20} 21$ The US Department of Health and Human Services' Biomedical Advanced Research and Development Authority (BARDA) has supported Achaogen for developing a broad spectrum antibiotic to treat bioterrorism threats from plague, tularaemia infections, and drug resistant pathogens. ${ }^{22}$ In the UK, the Wellcome Trust has developed a broad portfolio of antibiotic projects, providing grants for small firms with promising early stage novel chemistry through its seeding drug discovery programme as well as funding for translational research to bring innovative treatment technologies closer to market. ${ }^{23}$ In seeking greater collaboration between the public and private sectors, the European Commission's Innovative Medicine Initiative is considering antibiotic resistance as a topic for $2012 .{ }^{24}$ However, ensuring fair returns on these public and philanthropic investments requires that society share in the rewards.

\section{Sharing rewards}

The average antibiotic approved between 1990 and 1994 had an economic value to a pharmaceutical firm of $\$ 2.4$ bn over a 20 year product life cycle, substantially less than the $\$ 4$. 2 bn for central nervous system drugs and $\$ 3.7 \mathrm{bn}$ for cardiovascular drugs. ${ }^{25}$ In 2009, the worldwide sales of central nervous system drugs were still nearly double the value of antibacterial drug sales - and those of cardiovascular drugs were over three times the value. ${ }^{26-28}$ Over the past five years, the antibiotics market registered only $4 \%$ annual growth while antiviral drugs and vaccines exceeded $16 \% .^{29}$ Clearly antibiotics are less commercially attractive to companies than many other drugs, but any financial incentive to bring novel antibiotics to market must ensure that their use is safe, rational, and affordable to those in need.

Many incentives reliant on market exclusivity tie financial returns to sales rather than rational and affordable use. Some have sought to mitigate these shortcomings with proposals for broad patents over groups of antibiotics that compete for effectiveness ${ }^{30}$ and value based reimbursement that is dependent on meeting drug conservation targets. ${ }^{31}$ US legislative initiatives like the Generating Antibiotic Incentives Now (GAIN) Act unfortunately mainly use data exclusivity in the hope that extending the monopoly protection on novel antibiotics to treat multidrug resistant infections will give companies added 
incentive.$^{32}$ However, industry, at least in Europe, increasingly acknowledges the need to delink incentives from sales of the product. ${ }^{33}$ Proposals range from conditioning public funding with fair returns on research and development to buying out patents so that manufacturers can be licensed to produce antibiotics on a scale appropriate for rational use. For antibiotics, the existing imbalance between excess use and lack of access must also be addressed through optimal production volumes, controlled distribution, and rational use.

If public funds are invested in research and development it is fair to insist on sharing some of the rewards. Product development partnerships for antibiotics may ensure both fairer returns on public investment and more affordable pricing, as has been achieved by the Drugs for Neglected Diseases Initiative for antimalarial fixed dose combination drugs. ${ }^{34}$

\section{Conclusions}

The way forward will involve a dynamic mix of public-private partnership with solutions that tackle both scientific and financial bottlenecks in the pipeline. Sharing resources, risks, and rewards each suggest operating principles against which to benchmark potential solutions. For starters, sharing resources should extend the bounds for exploratory research and shift the line between precompetitive and competitive information. Sharing risks should extend public sector science and build infrastructure for collaborative research and development, and sharing rewards should delink financial returns from sales of the product and ensure fair returns for the public sharing of risks in investing in research and development. Importantly, the 3Rs should not be considered in isolation, but coordinated in an integrated approach. For example, resources from the NIH's National Center for Advancing Translational Sciences, the European Union's Innovative Medicines Initiative, and other funders might result in a coordinated platform for accelerating antibiotic innovation, both sharing resources and risks. Public sector capital invested in antibiotic innovation might be structured in a way to lower the costs of private sector capital investments and also ensure fair returns to the public. Finding the right strategic mix of approaches remains the challenge ahead.

Contributors and sources: As an officer at the Rockefeller Foundation, a member of the Institute of Medicine's Committee on Accelerating Rare Diseases Research and Orphan Product Development, and director of the Duke Program on Global Health and Technology Access, ADS has both conducted research and advised intergovernmental agencies and public-private partnerships on pharmaceutical innovation and access. $\mathrm{OC}$ has a long experience from preclinical and clinical antibiotic research and introduced the issue of the dearth of novel antibiotics as one of the important work areas for ReAct. Both ADS and OC were involved in the EMA-ECDC-ReAct pipeline analysis of novel antibiotics.

Competing interests: All authors have completed the ICMJE uniform disclosure form at www.icmje.org/coi_disclosure.pdf (available on request from the corresponding author) and declare: no support from any organisation for the submitted work and no financial relationships with any organisations that might have an interest in the submitted work in the previous three years. ADS directs the Strategic Policy Unit of ReAct-Action on Antibiotic Resistance, and was chair of the WHO/World Alliance for Patient Safety Working Group on Antimicrobial Research and Development. OC is the chairman and founding executive director of ReAct and served as co-chair of the WHO /World Alliance for Patient Safety Working Group on Rational Use of Antimicrobials.

Provenance and peer review: Commissioned; externally peer reviewed.

1 Spellberg B, Powers J, Brass E, Miller L, Edwards J. Trends in antimicrobial drug development: implications for the future. Clin Infect Dis 2004;38:1279-86.

2 Aronsson B, Boscan IS, Cars O, Giamarellou H, Gyssens IC, Lutsar I, et al. The bacterial challenge: time to react. European Centre for Disease Prevention and Control and European Medicines Agency joint report. 2009 www.ema.europa.eu/pdfs/human/ antimicrobial resistance/EMEA-576176-2009.pdf

3 Hamad B. The antibiotics market. Nature Rev Drug Discovery 2006;9:675-6.

4 Projan SJ. Why is big pharma getting out of antibacterial drug discovery? Curr Opin Microbiol 2003;6:427-30.

5 Mossialos E, Morel C, Edwards S, Berenson J, Gemmill-Toyama M, Brogan D. Policies and incentives to promote innovation in antibiotic research. Final report 2010. www.euro. who.int/_data/assets/pdf_file/0011/120143/E94241.

6 Kesselheim A, Outterson K. Fighting antibiotic resistance: marrying new financial incentives to meeting public health goals. Health Aff 2010;29:1689.

7 Payne D, Gwynn M, Holmes D, Pompliano D. Drugs for bad bugs: confronting the challenges of antibacterial discovery. Nature Rev Drug Discovery 2007;6:29-40.

8 Schmid M. Seeing is believing: the impact of structural genomics on antimicrobial drug discovery. Nature 2004;2:239.

9 ReAct. Collaboration for innovation-the urgent need for new antibiotics. ReAct policy seminar, 23 May 2011, Brussels, Belgium. www.reactgroup.org/uploads/news/ Collaboration-for-Innovation-The-Urgent-Need-for-New-Antibiotics-ReAct-Policy-SeminarBrussels-May-23-2011.pdf.

10 Bleicher K, Bohm H, Muller K, Alanine A. Hit and lead generation: beyond high-throughput screening. Nature Rev Drug Discovery 2003;2:369.

11 Fischer A, Borensztein P, Roussel C. The European rare diseases therapeutic initiative. PLOS Med 2005:2:e243.

12 Nwaka S, Ramirez B, Brun R, Maes L, Douglas F, Ridley R. Advancing drug innovation for neglected diseases—criteria for lead progression. PLoS Negl Trop Dis 2009;8:e440.

13 Rai AK, Reichman JH, Uhlir PF, Crossman C. Pathways across the valley of death: nove intellectual property strategies for accelerated drug discovery. Yale J Health Law, Policy, Ethics 2008;8:53-89.

14 Coalition Against Major Diseases. www.c-path.org/CAMD.cfm

15 Austin C, Brady L, Insel T, Collins F. NIH molecular libraries initiative. Science 2004;306:1138.

16 Collins F. Reengineering translational science: the time is right. Science Trans/ Med 2011;3:90:90cm17.www.nih.gov/about/director/articles/translational_science_07062011. pdf.

17 Goss C, Mayer-Hamblett N. The Cystic Fibrosis Foundation therapeutics development network: a national effort by the Cystic Fibrosis Foundation to build a clinical trials network. Children's Health Care 2008;37:5-20.

18 Ghosh S. How the young brigade mapped the TB genome. The Hindu 2010. http://beta thehindu.com/sci-tech/article397395.ece.

19 Nwaka S, llunga T, Da Silva J, Verde E, Hackley D, Vre R, et al. Developing ANDI: a novel approach to health product R\&D in Africa. PLoS Medicine 2010;7:e1000293.

20 GlaxoSmithKline. GlaxoSmithKline awarded US Department of Defense contract to pursue novel antibacterial research program. 2007. http://us.gsk.com/html/media-news/ pressreleases/2007/2007_09_18_GSK1146.htm.

21 Fikes B. Trius receives $\$ 29.5$ million contract to find microbe antibiotics. North County Times 2010 Sep 20. www.nctimes.com/business/article_bb6141ed-f20b-5016-b00e96a2bacab749.html.

$22 \mathrm{http}: / /$ /US Department of Health \& Human Services. BARDA funds drug development for biothreats, antibiotic resistance. Press release, 30 August 2010. www.hhs.gov/news/press/ 2010pres/08/20100830a.html.

23 Wellcome Trust. Therapeutics. 2010. www.wellcome.ac.uk/Funding/Technology-transfer/ Funded-projects/therapeutics/index.htm.

24 Innovative Medicines Initiative. NewDrugs4BadBugs (ND4BB). www.imi.europa.eu/content/ future-topics.

25 DiMasi J, Grabowski H, Vernon J. R\&D costs and returns by therapeutic category. Drug Info J 2004;38:211-23.

26 BCC Research. Infectious disease treatments: global markets. 2010. www.bccresearch. com/report/PHM061A.html.

27 BCC Research. Therapeutic drugs for central nervous system (CNS) disorders: technologies and global markets. 2010. www.bccresearch.com/report/PHM068A.html.

28 BCC Research. Cardiovascular therapeutic drugs: technologies and global markets. 2010. www.bccresearch.com/report/cardiovascular-therapeutic-drugs-phm024b.html.

29 Hamad B. The antibiotics market. Nature Rev Drug Discovery 2010;9:675-6.

30 Laxminarayan R. Antibiotic resistance: can economic incentives play a role? 2001. www. rff.org/RFF/Documents/RFF-Resources-143-antibiotic.pdf.

31 Kesselheim A, Outterson K. Fighting antibiotic resistance: marrying new financial incentives to meeting public health goals. Health Aff 2010;29:1689.

32 Govtrack.us. Text of H.R. 2182: Generating Antibiotic Incentives Now Act of 2011. www. govtrack.us/congress/billtext.xpd?bill=h112-2182.

33 Bergström $\mathrm{R}$. The role of the pharmaceutical industry in meeting the public health threat of antibacterial resistance. Drug Resist Updates 2010;14:77-8.

34 ASAQ: Hope for Malaria. New, once-a-day fixed-dose combination against malaria now available. Press release, 1 March 2007. www.actwithasaq.org/en/press_2.htm. 


\section{Figure}

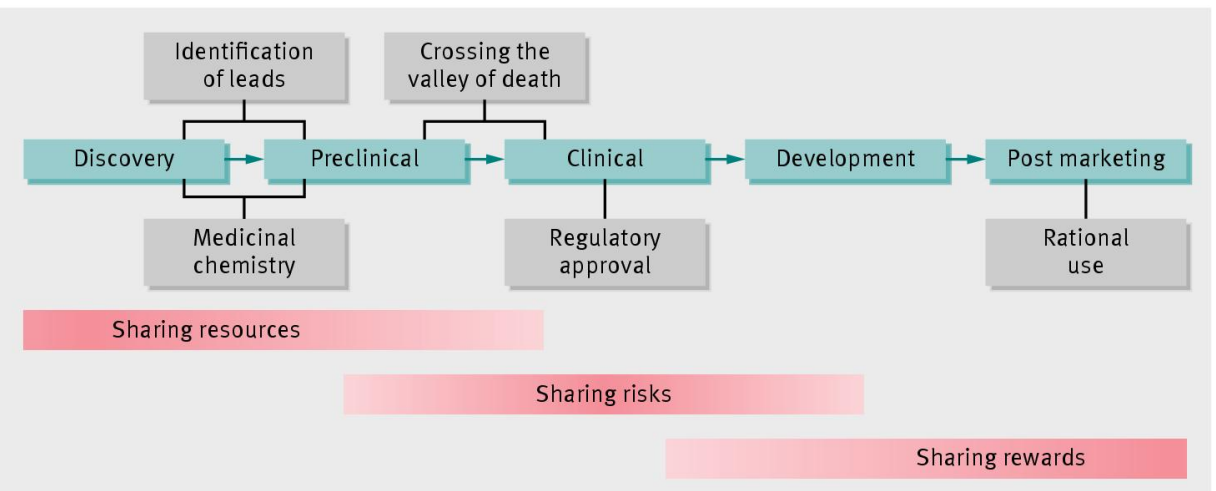

Bottlenecks in the antibiotic pipeline 